\title{
A DENSITY FUNCTIONAL THEORY STUDY BASED ON MONOLIGNOLS: MOLECULAR STRUCTURE, HOMO-LUMO ANALYSIS, MOLECULAR ELECTROSTATIC POTENTIAL
}

\author{
FERIDE AKMAN \\ University of Bingöl, Vocational School of Technical Sciences, Bingöl 12000, Turkey \\ \Corresponding author: chemakman@gmail.com
}

Received May 15, 2018

In the present study, three monomeric subunits, sinapyl alcohol, coniferyl alcohol and p-coumaryl alcohol, were investigated in terms of chemical shift, vibrational spectroscopic, molecular geometry and quantum chemical calculations. HOMO-LUMO analysis, electronegativity, hardness, electrophilicity index, softness and molecular electrostatic potential (MEP) results were also reported using the density functional theory (DFT/B3LYP) method with 6-31G (d,p) basis set. The computed HOMO and LUMO energies confirmed that charge transfer took place within the monolignols. The positive region of the MEP is associated with nucleophilic reactivity and the negative region - with electrophilic reactivity, as shown in the MEP plots, while the monolignols have various probable regions.

Keywords: lignin, density functional theory (DFT), coniferyl alcohol, p-coumaryl alcohol, sinapyl alcohol

\section{INTRODUCTION}

Lignin is the second most abundant terrestrial biopolymer, or renewable natural polymer, after cellulose. It has various properties and applications, e.g. as adhesive, binder, fiber nutritional source, dispersing agent, flocculent and thickener in paints and coatings, which can increase its potential value. Lignin also provides defense against pathogens and pests and is very important for both mechanical and vascular transport. ${ }^{1,2}$

Lignin has a complex three-dimensional structure formed by free radical polymerization of three monomeric subunits, i.e. sinapyl alcohol, pcoumaryl alcohol and coniferyl alcohol, in plants. ${ }^{3}$ These precursors are called monolignols. Based on the type, part and growth environment of the plant, its lignin structure may differ in terms of the amount and type of monomeric subunits. $^{4,5}$ For instance, hardwood lignin involves approximately 1:1 (sinapyl:coniferyl alcohol) monomeric units, whereas softwood lignin contains these units in a roughly 1:9 ratio. ${ }^{6}$ Moreover, lignin is one of the primary cell wall ingredients in arboreal plants and, after cellulose, it is the best known biopolymer. Apart from its structural function, lignin plays a very important role biologically in plants. Besides, lignin prevents the absorption of water by polysaccharides, as it is much less hydrophilic than hemicelluloses and cellulose. Therefore, knowledge of its chemical structure is as important as that of other natural polymers. In the industry, only a small fraction of the lignin obtained as a waste by-product is used commercially, thus valorizing its various aromatic units, functional groups, conjugated linkages and molecular size. ${ }^{7}$ Therefore, a detailed investigation of lignin and its components could provide solutions to the problem. While such a study would be expensive and time-consuming, a theoretical approach will bring both time and cost reducing benefits. The structure and reactivity of lignin can be researched by theoretical methods. Recently, the density functional theory (DFT), which is one of the most important theoretical modeling methods, has been used to predict correctly the structures, as well as the physical and chemical properties of molecules. ${ }^{8,9}$

The aim of this study was to obtain deeper understanding of the three monomeric subunits of lignin using the density functional theory (DFT) method. Density functional theory (DFT) calculations were performed for the monolignols, using the standard 6-31G $(\mathrm{d}, \mathrm{p})$ basis set. 
The FT-IR and ${ }^{1} \mathrm{H}-\mathrm{NMR}$ of the monolignols were determined at the DFT/B3LYP level of the theory using the 6-31G $(\mathrm{d}, \mathrm{p})$ basis set. The data calculated for monolignols are very important for providing an insight into molecular analysis and then for the use of lignin in technological applications. Considering the fact that the studies using the DFT method, conducted in the past and present, have been confirmed experimentally, future investigations using this method can provide a great guide to chemists and physicists, without requiring experimental studies.

\section{EXPERIMENTAL}

\section{Computational methods}

The molecular structures of monolignols in the ground state were optimized, using the Gaussian $09^{10}$ and Gaussview 5.0.9 molecular visualization package program ${ }^{11}$ the hybrid functional and Becke's three parameter functional (B3), ${ }^{12}$ combined with the gradient-corrected correlational functional of Lee, Yang and Parr (LYP), ${ }^{13,14}$ supplemented with the standard 6-31G $(\mathrm{d}, \mathrm{p})$ basis set. The ${ }^{1} \mathrm{H}$ chemical shifts, vibrational frequencies, HOMO-LUMO energies, and molecular electrostatic potential surfaces of monolignols were calculated by the same method.

\section{RESULTS AND DISCUSSION Molecular geometry}

Molecular geometry optimizations of the monolignols were performed by the 6-31G $(\mathrm{d}, \mathrm{p})$ basis set and indicated in Figure 1 with a numbering scheme. The minimum self- consistence field energy for sinapyl, coniferyl and p-coumaryl alcohols was found to be -19822.114, -16705.456 and -13589.425 , respectively. The geometrical parameters optimized for monolignols are listed in Table 1, along with the atomic labelling indicated in Figure 1. The longest lengths for sinapyl, coniferyl and pcoumaryl alcohols are between the $\mathrm{C} 1-\mathrm{C} 2$, C9H19 and H14-H19 bond distances and indicate values of $1.5064,2.7064$ and $2.1837 \AA$, respectively, whereas the shortest lengths are $\mathrm{O} 15-\mathrm{H} 29, \quad \mathrm{O} 13-\mathrm{H} 25$ and $\mathrm{O} 11-\mathrm{H} 21$ bond distances and indicate values of $0.9650,0.9637$ and $0.9637 \AA$, respectively. Comparing bond lengths, the $\mathrm{C}-\mathrm{H}$ and $\mathrm{C}-\mathrm{C}$ bond lengths are longer than the $\mathrm{O}-\mathrm{H}$ bond lengths due to the electronegativity of Oxygen (O). In sinapyl alcohol, one of the largest bond angles is computed as $127.622^{\circ}$ and ascribed to C2-C3$\mathrm{C} 4$, while one of the smallest bond angles is computed as $104.9627^{\circ}$ and ascribed to O7-C8$\mathrm{H} 21$. In coniferyl alcohol, one of the largest bond angles is computed as $168.273^{\circ}$ and ascribed to C11-C9-H19, while one of the smallest bond angles is computed as $72.766^{\circ}$ and ascribed to C6-C9-H19. In p-coumaryl alcohol, one of the largest bond angles is computed as $128.064^{\circ}$ and ascribed to $\mathrm{C} 2-\mathrm{C} 3-\mathrm{C} 4$, while one of the smallest bond angles is computed as $106.444^{\circ}$ and ascribed to $\mathrm{H} 12-\mathrm{C} 1-\mathrm{H} 13$.
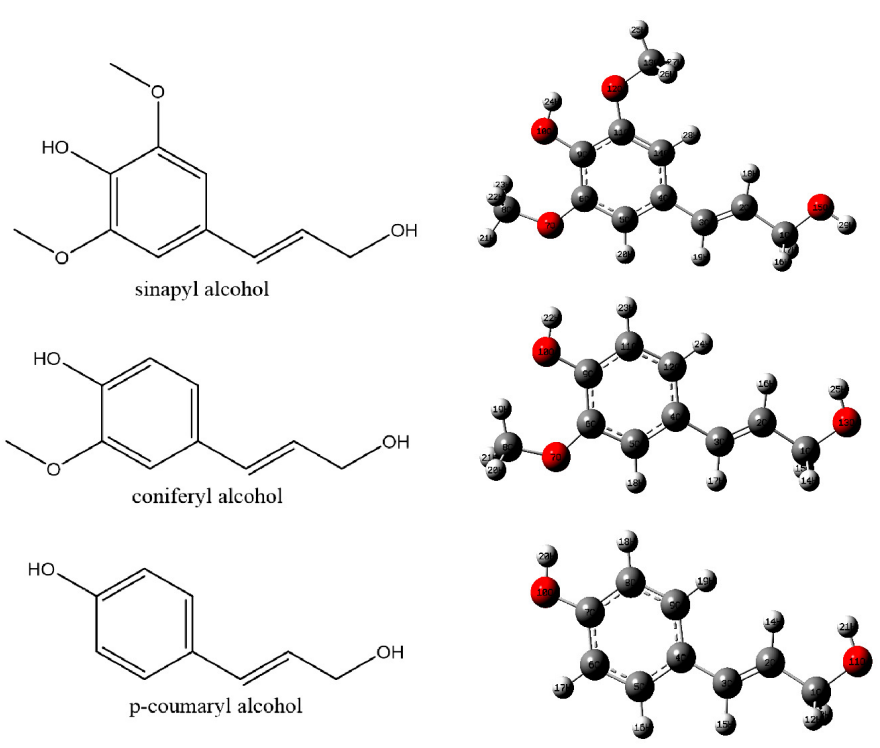

Figure 1: Optimized molecular structure of monolignols 
Table 1

Optimized bond lengths $(\AA)$, bond angles $\left({ }^{\circ}\right)$, dihedral angles $\left({ }^{\circ}\right)$ of monolignols at B3LYP/ 6-31G $(\mathrm{d}, \mathrm{p})$ basis set in gaseous phase

\begin{tabular}{|c|c|c|c|c|c|}
\hline Parameters & Sinapyl alcohol & Parameters & Coniferyl alcohol & Parameters & p-Coumaryl alcohol \\
\hline \multicolumn{6}{|c|}{ Bond lengths $(\AA)$} \\
\hline $\mathrm{C} 1-\mathrm{C} 2$ & 1.5064 & C1-C2 & 1.5119 & $\mathrm{C} 1-\mathrm{C} 2$ & 1.5115 \\
\hline C1-O15 & 1.4243 & $\mathrm{C} 1-\mathrm{O} 13$ & 1.4232 & C1-O11 & 1.424 \\
\hline C1-H16 & 1.1035 & C1-H14 & 1.101 & C1-H12 & 1.1011 \\
\hline C1-H17 & 1.1035 & C1-H15 & 1.101 & $\mathrm{C} 1-\mathrm{H} 13$ & 1.1011 \\
\hline C2-C3 & 1.3395 & $\mathrm{C} 2-\mathrm{C} 3$ & 1.3398 & $\mathrm{C} 2-\mathrm{C} 3$ & 1.34 \\
\hline C2-H18 & 1.0877 & C2-H16 & 1.0916 & C2-H14 & 1.0916 \\
\hline C3-C4 & 1.4689 & $\mathrm{C} 3-\mathrm{C} 4$ & 1.4691 & $\mathrm{C} 3-\mathrm{C} 4$ & 1.4682 \\
\hline C3-H19 & 1.0911 & C3-H17 & 1.0909 & C3-H15 & 1.0912 \\
\hline C4-C5 & 1.3959 & C4-C5 & 1.4002 & $\mathrm{C} 4-\mathrm{C} 5$ & 1.4072 \\
\hline C4-C14 & 1.4106 & C4-C12 & 1.4054 & C4-C9 & 1.4063 \\
\hline C5-C6 & 1.4035 & C5-C6 & 1.4011 & C5-C6 & 1.3898 \\
\hline $\mathrm{C} 5-\mathrm{H} 20$ & 1.0852 & C5-H18 & 1.0857 & C5-H16 & 1.087 \\
\hline C6-O7 & 1.3656 & C6-O7 & 1.3627 & C6-C7 & 1.398 \\
\hline C6-C9 & 1.4007 & C6-C9 & 1.411 & C6-H17 & 1.0848 \\
\hline $\mathrm{O} 7-\mathrm{C} 8$ & 1.4268 & $\mathrm{O} 7-\mathrm{C} 8$ & 1.4306 & $\mathrm{C} 7-\mathrm{C} 8$ & 1.4008 \\
\hline $\mathrm{C} 8-\mathrm{H} 21$ & 1.092 & C8-H19 & 1.0853 & C7-O10 & 1.3654 \\
\hline C8-H22 & 1.0933 & $\mathrm{C} 8-\mathrm{H} 20$ & 1.0951 & C8-C9 & 1.39 \\
\hline $\mathrm{C} 8-\mathrm{H} 23$ & 1.0933 & $\mathrm{C} 8-\mathrm{H} 21$ & 1.0951 & C8-H18 & 1.0882 \\
\hline C9-O10 & 1.3709 & C9-O10 & 1.377 & C9-H19 & 1.0856 \\
\hline C9-C11 & 1.4132 & C9-C11 & 1.4004 & $\mathrm{O} 10-\mathrm{H} 20$ & 0.9662 \\
\hline O10-H24 & 0.9704 & C9-H19 & 2.7064 & $\mathrm{O} 11-\mathrm{H} 21$ & 0.9637 \\
\hline C11-O12 & 1.3781 & O10-H19 & 2.0545 & H14-H19 & 2.1837 \\
\hline C11-C14 & 1.385 & $\mathrm{O} 10-\mathrm{H} 22$ & 0.966 & & \\
\hline O12-C13 & 1.4197 & $\mathrm{C} 11-\mathrm{C} 12$ & 1.3869 & & \\
\hline $\mathrm{C} 13-\mathrm{H} 25$ & 1.0909 & $\mathrm{C} 11-\mathrm{H} 23$ & 1.0891 & & \\
\hline C13-H26 & 1.0967 & $\mathrm{C} 12-\mathrm{H} 24$ & 1.0846 & & \\
\hline C13-H27 & 1.0967 & O13-H25 & 0.9637 & & \\
\hline C14-H28 & 1.0821 & H16-H24 & 2.1927 & & \\
\hline $\mathrm{O} 15-\mathrm{H} 29$ & 0.965 & & & & \\
\hline \multicolumn{6}{|c|}{ Bond angles $\left({ }^{\circ}\right)$} \\
\hline $\mathrm{C} 2-\mathrm{C} 1-\mathrm{O} 15$ & 107.6851 & C2-C1-O13 & 114.06 & $\mathrm{C} 2-\mathrm{C} 1-\mathrm{O} 11$ & 114.0588 \\
\hline C2-C1-H16 & 110.2064 & $\mathrm{C} 2-\mathrm{C} 1-\mathrm{H} 14$ & 109.7407 & $\mathrm{C} 2-\mathrm{C} 1-\mathrm{H} 12$ & 109.774 \\
\hline C2-C1-H17 & 110.2065 & C2-C1-H15 & 109.7407 & $\mathrm{C} 2-\mathrm{C} 1-\mathrm{H} 13$ & 109.774 \\
\hline O15-C1-H16 & 111.0208 & O13-C1-H14 & 108.278 & O11-C1-H12 & 108.2484 \\
\hline O15-C1-H17 & 111.0217 & O13-C1-H15 & 108.278 & O11-C1-H13 & 108.2484 \\
\hline H16-C1-H17 & 106.7235 & H14-C1-H15 & 106.4522 & H12-C1-H13 & 106.444 \\
\hline C1-C2-C3 & 124.3961 & C1-C2-C3 & 124.4149 & C1-C2-C3 & 124.3921 \\
\hline C1-C2-H18 & 113.9402 & $\mathrm{C} 1-\mathrm{C} 2-\mathrm{H} 16$ & 115.8405 & $\mathrm{C} 1-\mathrm{C} 2-\mathrm{H} 14$ & 115.7999 \\
\hline C3-C2-H18 & 121.6636 & C3-C2-H16 & 119.7446 & C3-C2-H14 & 119.808 \\
\hline C2-C3-C4 & 127.6226 & C2-C3-C4 & 127.7489 & C2-C3-C4 & 128.0643 \\
\hline C2-C3-H19 & 118.1457 & C2-C3-H17 & 117.8309 & C2-C3-H15 & 117.6428 \\
\hline C4-C3-H19 & 114.2317 & C4-C3-H17 & 114.4201 & C4-C3-H15 & 114.2929 \\
\hline C3-C4-C5 & 118.7521 & C3-C4-C5 & 118.6744 & C3-C4-C5 & 119.1134 \\
\hline C3-C4-C14 & 122.7681 & C3-C4-C12 & 123.7858 & C3-C4-C9 & 123.6661 \\
\hline C5-C4-C14 & 118.4798 & $\mathrm{C} 5-\mathrm{C} 4-\mathrm{C} 12$ & 117.5399 & C5-C4-C9 & 117.2205 \\
\hline C4-C5-C6 & 122.6388 & C4-C5-C6 & 123.5677 & $\mathrm{C} 4-\mathrm{C} 5-\mathrm{C} 6$ & 122.0277 \\
\hline C4-C5-H20 & 120.5219 & $\mathrm{C} 4-\mathrm{C} 5-\mathrm{H} 18$ & 120.1614 & C4-C5-H16 & 119.0049 \\
\hline C6-C5-H20 & 116.8393 & C6-C5-H18 & 116.2709 & C6-C5-H16 & 118.9674 \\
\hline C5-C6-O7 & 114.3496 & C5-C6-O7 & 113.5002 & C5-C6-C7 & 119.628 \\
\hline C5-C6-C9 & 118.712 & C5-C6-C9 & 117.8002 & C5-C6-H17 & 121.3416 \\
\hline O7-C6-C9 & 126.9384 & O7-C6-C9 & 128.6996 & C7-C6-H17 & 119.0304 \\
\hline C6-O7-C8 & 121.8917 & C6-O7-C8 & 125.67 & C6-C7-C8 & 119.5271 \\
\hline O7-C8-H21 & 104.9627 & O7-C8-H19 & 113.2437 & C6-C7-O10 & 117.6993 \\
\hline $\mathrm{O} 7-\mathrm{C} 8-\mathrm{H} 22$ & 111.8518 & $\mathrm{O} 7-\mathrm{C} 8-\mathrm{H} 20$ & 107.7553 & C8-C7-O10 & 122.7736 \\
\hline $\mathrm{O} 7-\mathrm{C} 8-\mathrm{H} 23$ & 111.8517 & O7-C8-H21 & 107.7553 & C7-C8-C9 & 120.1723 \\
\hline H21-C8-H22 & 109.6011 & H19-C8-H20 & 109.6561 & C7-C8-H18 & 119.8862 \\
\hline H21-C8-H23 & 109.6009 & H19-C8-H21 & 109.6561 & C9-C8-H18 & 119.9415 \\
\hline $\mathrm{H} 22-\mathrm{C} 8-\mathrm{H} 23$ & 108.8938 & $\mathrm{H} 20-\mathrm{C} 8-\mathrm{H} 21$ & 108.6545 & C4-C9-C8 & 121.4244 \\
\hline C6-C9-O10 & 122.733 & C6-C9-O10 & 120.6374 & C4-C9-H19 & 120.0678 \\
\hline C6-C9-C11 & 118.6084 & С6-C9-C11 & 118.9599 & С8-C9-H19 & 118.5079 \\
\hline O10-C9-C11 & 118.6586 & C6-C9-H19 & 72.7666 & C7-O10-H20 & 109.1721 \\
\hline C9-O10-H24 & 105.8754 & O10-C9-C11 & 120.4026 & $\mathrm{C} 1-\mathrm{O} 11-\mathrm{H} 21$ & 108.5672 \\
\hline C9-C11-O12 & 112.2267 & C11-C9-H19 & 168.2735 & C2-H14-H19 & 114.1678 \\
\hline C9-C11-C14 & 122.3739 & C9-O10-H22 & 108.3539 & C9-H19-H14 & 114.226 \\
\hline O12-C11-C14 & 125.3994 & H19-O10-H22 & 149.3222 & & \\
\hline
\end{tabular}




\section{FERIDE AKMAN}

\begin{tabular}{|c|c|c|c|c|c|}
\hline \\
\hline C11-O12-C13 & 118.2896 & $\mathrm{C} 9-\mathrm{C} 11-\mathrm{C} 12$ & 122.3225 & & \\
\hline $\mathrm{O} 12-\mathrm{C} 13-\mathrm{H} 25$ & 106.1966 & C9-C11-H23 & 118.0535 & & \\
\hline O12-C13-H26 & 111.2969 & $\mathrm{C} 12-\mathrm{C} 11-\mathrm{H} 23$ & 119.624 & & \\
\hline $\mathrm{O} 12-\mathrm{C} 13-\mathrm{H} 27$ & 111.2968 & C4-C12-C11 & 119.8098 & & \\
\hline $\mathrm{H} 25-\mathrm{C} 13-\mathrm{H} 26$ & 109.3956 & $\mathrm{C} 4-\mathrm{C} 12-\mathrm{H} 24$ & 121.0154 & & \\
\hline H25-C13-H27 & 109.3958 & $\mathrm{C} 11-\mathrm{C} 12-\mathrm{H} 24$ & 119.1748 & & \\
\hline $\mathrm{H} 26-\mathrm{C} 13-\mathrm{H} 27$ & 109.1962 & $\mathrm{C} 1-\mathrm{O} 13-\mathrm{H} 25$ & 108.5763 & & \\
\hline C4-C14-C11 & 119.187 & C2-H16-H24 & 114.6406 & & \\
\hline C4-C14-H28 & 120.3706 & C8-H19-C9 & 99.6202 & & \\
\hline C11-C14-H28 & 120.4425 & C8-H19-O10 & 129.4254 & & \\
\hline C1-O15-H29 & 107.9051 & C12-H24-H16 & 113.0646 & & \\
\hline \multicolumn{6}{|c|}{ Dihedral angles $\left(^{\circ}\right)$} \\
\hline O15-C1-C2-C3 & 179.9768 & O13-C1-C2-C3 & 180 & O11-C1-C2-C3 & 180 \\
\hline O15-C1-C2-H18 & -0.0248 & $\mathrm{O} 13-\mathrm{C} 1-\mathrm{C} 2-\mathrm{H} 16$ & 0 & $\mathrm{O} 11-\mathrm{C} 1-\mathrm{C} 2-\mathrm{H} 14$ & 0 \\
\hline H16-C1-C2-C3 & -58.7883 & H14-C1-C2-C3 & -58.3226 & H12-C1-C2-C3 & -58.337 \\
\hline $\mathrm{H} 16-\mathrm{C} 1-\mathrm{C} 2-\mathrm{H} 18$ & 121.2101 & H14- C1-C2-H16 & 121.6774 & $\mathrm{H} 12-\mathrm{C} 1-\mathrm{C} 2-\mathrm{H} 14$ & 121.663 \\
\hline H17-C1-C2-C3 & 58.7408 & H15-C1-C2-C3 & 58.3226 & H13-C1-C2-C3 & 58.337 \\
\hline $\mathrm{H} 17-\mathrm{C} 1-\mathrm{C} 2-\mathrm{H} 18$ & -121.2608 & $\mathrm{H} 15-\mathrm{C} 1-\mathrm{C} 2-\mathrm{H} 16$ & -121.6774 & $\mathrm{H} 13-\mathrm{C} 1-\mathrm{C} 2-\mathrm{H} 14$ & -121.663 \\
\hline $\mathrm{C} 2-\mathrm{C} 1-\mathrm{O} 15-\mathrm{H} 29$ & -179.9931 & $\mathrm{C} 2-\mathrm{C} 1-\mathrm{O} 13-\mathrm{H} 25$ & 0 & $\mathrm{C} 2-\mathrm{C} 1-\mathrm{O} 11-\mathrm{H} 21$ & 0 \\
\hline H16-C1-O15-H29 & 59.2823 & $\mathrm{H} 14-\mathrm{C} 1-\mathrm{O} 13-\mathrm{H} 25$ & -122.4814 & $\mathrm{H} 12-\mathrm{C} 1-\mathrm{O} 11-\mathrm{H} 21$ & -122.5016 \\
\hline H17-C1-O15-H29 & -59.2678 & $\mathrm{H} 15-\mathrm{C} 1-\mathrm{O} 13-\mathrm{H} 25$ & 122.4814 & H13-C1-O11-H21 & 122.5016 \\
\hline $\mathrm{C} 1-\mathrm{C} 2-\mathrm{C} 3-\mathrm{C} 4$ & 179.9974 & C1-C2-C3-C4 & 180 & C1-C2-C3-C4 & 180 \\
\hline C1-C2-C3-H19 & -0.003 & C1-C2-C3-H17 & 0 & C1-C2-C3-H15 & 0 \\
\hline H18-C2-C3-C4 & $-9 \mathrm{E}-4$ & H16-C2-C3-C4 & 0 & H14-C2-C3-C4 & 0 \\
\hline H18-C2-C3-H19 & 179.9987 & $\mathrm{H} 16-\mathrm{C} 2-\mathrm{C} 3-\mathrm{H} 17$ & 180 & $\mathrm{H} 14-\mathrm{C} 2-\mathrm{C} 3-\mathrm{H} 15$ & 180 \\
\hline C2-C3-C4-C5 & 179.9973 & $\mathrm{C} 1-\mathrm{C} 2-\mathrm{H} 16-\mathrm{H} 24$ & 180 & C1-C2-H14-H19 & 180 \\
\hline C2-C3-C4-C14 & -0.0025 & $\mathrm{C} 3-\mathrm{C} 2-\mathrm{H} 16-\mathrm{H} 24$ & 0 & DC3-C2-H14-H19 & 0 \\
\hline $\mathrm{H} 19-\mathrm{C} 3-\mathrm{C} 4-\mathrm{C} 5$ & -0.0024 & $\mathrm{C} 2-\mathrm{C} 3-\mathrm{C} 4-\mathrm{C} 5$ & 180 & C2-C3-C4-C5 & 180 \\
\hline H19-C3-C4-C14 & 179.9978 & C2-C3-C4-C12 & 0 & C2-C3-C4-C9 & 0 \\
\hline C3-C4-C5-C6 & -179.9997 & $\mathrm{H} 17-\mathrm{C} 3-\mathrm{C} 4-\mathrm{C} 5$ & 0 & $\mathrm{H} 15-\mathrm{C} 3-\mathrm{C} 4-\mathrm{C} 5$ & 0 \\
\hline C3-C4-C5-H20 & 0 & $\mathrm{H} 17-\mathrm{C} 3-\mathrm{C} 4-\mathrm{C} 12$ & 180 & $\mathrm{H} 15-\mathrm{C} 3-\mathrm{C} 4-\mathrm{C} 9$ & 180 \\
\hline C14-C4-C5-C6 & 1E-4 & C3-C4-C5-C6 & 180 & C3-C4-C5-C6 & 180 \\
\hline C14-C4-C5-H20 & 179.9998 & C3-C4-C5-H18 & 0 & C3-C4-C5-H16 & 0 \\
\hline C3-C4-C14-C11 & 179.9996 & C12-C4-C5-C6 & 0 & C9-C4-C5-C6 & 0 \\
\hline $\mathrm{C} 3-\mathrm{C} 4-\mathrm{C} 14-\mathrm{H} 28$ & $-9 \mathrm{E}-4$ & $\mathrm{C} 12-\mathrm{C} 4-\mathrm{C} 5-\mathrm{H} 18$ & 180 & C9-C4-C5-H16 & 180 \\
\hline C5-C4-C14-C11 & $-2 \mathrm{E}-4$ & $\mathrm{C} 3-\mathrm{C} 4-\mathrm{C} 12-\mathrm{C} 11$ & 180 & C3-C4-C9-C8 & 180 \\
\hline C5-C4-C14-H28 & 179.9994 & C3-C4-C12-H24 & 0 & C3-C4-C9-H19 & 0 \\
\hline C4-C5-C6-O7 & -179.9998 & $\mathrm{C} 5-\mathrm{C} 4-\mathrm{C} 12-\mathrm{C} 11$ & 0 & C5-C4-C9-C8 & 0 \\
\hline C4-C5-C6-C9 & $-2 \mathrm{E}-4$ & $\mathrm{C} 5-\mathrm{C} 4-\mathrm{C} 12-\mathrm{H} 24$ & 180 & C5-C4-C9-H19 & 180 \\
\hline $\mathrm{H} 20-\mathrm{C} 5-\mathrm{C} 6-\mathrm{O} 7$ & $5 \mathrm{E}-4$ & C4-C5-C6-O7 & 180 & C4-C5-C6-C7 & 0 \\
\hline $\mathrm{H} 20-\mathrm{C} 5-\mathrm{C} 6-\mathrm{C} 9$ & -179.9999 & C4-C5-C6-C9 & 0 & C4-C5-C6-H17 & 180 \\
\hline C5-C6-O7-C8 & -179.9912 & H18-C5-C6-O7 & 0 & H16-C5-C6-C7 & 180 \\
\hline C9-C6-O7-C8 & 0.0092 & H18-C5-C6-C9 & 180 & H16-C5-C6-H17 & 0 \\
\hline C5-C6-C9-O10 & -179.9992 & C5-C6-O7-C8 & 180 & C5-C6-C7-C8 & 0 \\
\hline C5-C6-C9-C11 & 3E-4 & C9-C6-O7-C8 & 0 & C5-C6-C7-O10 & 180 \\
\hline O7-C6-C9-O10 & 3E-4 & C5-C6-C9-O10 & 180 & H17-C6-C7-C8 & 180 \\
\hline O7-C6-C9-C11 & 179.9998 & C5-C6-C9-C11 & 0 & $\mathrm{H} 17-\mathrm{C} 6-\mathrm{C} 7-\mathrm{O} 10$ & 0 \\
\hline C6-O7-C8-H21 & 179.995 & С5-C6-C9-H19 & 180 & C6-C7-C8-C9 & 0 \\
\hline C6-O7-C8-H22 & -61.2348 & O7-C6-C9-O10 & 0 & C6-C7-C8-H18 & 180 \\
\hline C6-O7-C8-H23 & 61.225 & O7-C6-C9-C11 & 180 & $\mathrm{O} 10-\mathrm{C} 7-\mathrm{C} 8-\mathrm{C} 9$ & 180 \\
\hline C6-C9-O10-H24 & -179.9992 & O7-C6-C9-H19 & 0 & O10-C7-C8-H18 & 0 \\
\hline C11-C9-O10-H24 & 0.0012 & C6-O7-C8-H19 & 0 & C6-C7-O10-H20 & 180 \\
\hline C6-C9-C11-O12 & -179.9993 & $\mathrm{C} 6-\mathrm{O} 7-\mathrm{C} 8-\mathrm{H} 20$ & -121.4615 & C8-C7-O10-H20 & 0 \\
\hline C6-C9-C11-C14 & $-4 \mathrm{E}-4$ & C6-O7-C8-H21 & 121.4615 & C7-C8-C9-C4 & 0 \\
\hline O10-C9-C11-O12 & 2E-4 & O7-C8-H19-C9 & 0 & C7-C8-C9-H19 & 180 \\
\hline O10-C9-C11-C14 & 179.9992 & $\mathrm{O} 7-\mathrm{C} 8-\mathrm{H} 19-\mathrm{O} 10$ & 0 & $\mathrm{H} 18-\mathrm{C} 8-\mathrm{C} 9-\mathrm{C} 4$ & 180 \\
\hline C9-C11-O12-C13 & -179.995 & H20-C8-H19-C9 & 120.3871 & H18-C8-C9-H19 & 0 \\
\hline C14-C11-O12-C13 & 0.0061 & $\mathrm{H} 20-\mathrm{C} 8-\mathrm{H} 19-\mathrm{O} 10$ & 120.3871 & C4-C9-H19-H14 & 0 \\
\hline C9-C11-C14-C4 & 3E-4 & H21-C8-H19-C9 & -120.3871 & C8-C9-H19-H14 & 180 \\
\hline C9-C11-C14-H28 & -179.9992 & H21-C8-H19-O10 & -120.3871 & C2-H14-H19-C9 & 0 \\
\hline O12-C11-C14-C4 & 179.9991 & C6-C9-O10-H22 & 180 & & \\
\hline O12-C11-C14-H28 & $-4 \mathrm{E}-4$ & C11-C9-O10-H22 & 0 & & \\
\hline $\mathrm{D}(\mathrm{C} 11-\mathrm{O} 12-\mathrm{C} 13-\mathrm{H} 25)$ & 179.9958 & C6-C9-C11-C12 & 0 & & \\
\hline C11-O12-C13-H26 & -61.0317 & C6-C9-C11-H23 & 180 & & \\
\hline \multirow[t]{10}{*}{ C11-O12-C13-H27 } & 61.0231 & O10-C9-C11-C12 & 180 & & \\
\hline & & O10-C9-C11-H23 & 0 & & \\
\hline & & H19-C9-C11-C12 & 180 & & \\
\hline & & $\mathrm{H} 19-\mathrm{C} 9-\mathrm{C} 11-\mathrm{H} 23$ & 0 & & \\
\hline & & C6-C9-H19-C8 & 0 & & \\
\hline & & C11-C9-H19-C8 & 180 & & \\
\hline & & H22-O10-H19-C8 & 180 & & \\
\hline & & C9-C11-C12-C4 & 0 & & \\
\hline & & C9-C11-C12-H24 & 180 & & \\
\hline & & $\mathrm{H} 23-\mathrm{C} 11-\mathrm{C} 12-\mathrm{C} 4$ & 180 & & \\
\hline
\end{tabular}




\begin{tabular}{cc}
$\mathrm{H} 23-\mathrm{C} 11-\mathrm{C} 12-\mathrm{H} 24$ & 0 \\
C4-C12-H24-H16 & 0 \\
C11-C12-H24-H16 & 180 \\
C2-H16-H24-C12 & 0 \\
\hline
\end{tabular}

\section{Vibrational frequencies}

The vibrational frequencies for the monolignols were computed by the DFT method with the 6-31G $(\mathrm{d}, \mathrm{p})$ basis set. The theoretical Fourier transform infrared spectra of sinapyl, coniferyl and p-coumaryl alcohols are illustrated in Figure 2, respectively. The aromatic $\mathrm{C}-\mathrm{H}$ stretching vibrations are generally found in the region of $3100-3000 \mathrm{~cm}^{-1} .{ }^{15}$ The vibrations related to the aromatic $\mathrm{C}-\mathrm{H}$ stretching for sinapyl, coniferyl and p-coumaryl alcohols were computed by the DFT in the vicinity of $3079-3105 \mathrm{~cm}^{-1}$, 3030-3082 $\mathrm{cm}^{-1}$ and 3040-3089 $\mathrm{cm}^{-1}$, respectively. The $\mathrm{O}-\mathrm{H}$ stretching vibrations are generally found in the region of $3400-3600 \mathrm{~cm}^{-1} .{ }^{16}$
The $\mathrm{O}-\mathrm{H}$ stretching vibrations bound to the aromatic ring were computed in the vicinity of 3607,3673 and $3674 \mathrm{~cm}^{-1}$, while the $\mathrm{O}-\mathrm{H}$ stretching vibrations bound to the ethylene group were calculated to be located in the vicinity of 3684, 3698 and $3699 \mathrm{~cm}^{-1}$, respectively. The vibrations of $=\mathrm{C}-\mathrm{H}$ stretching were calculated to lie in the regions of $3008-3055 \mathrm{~cm}^{-1}, 3004-3013$ $\mathrm{cm}^{-1}$ and 3001-3009 $\mathrm{cm}^{-1}$, respectively. The calculated aliphatic $\mathrm{C}=\mathrm{C}$ stretching vibrations were found at 1662,1658 and $1654 \mathrm{~cm}^{-1}$, while the calculated aromatic $\mathrm{C}=\mathrm{C}$ stretching vibrations were found around 1489-1594, 1501-1598 and $1500-1606 \mathrm{~cm}^{-1}$, respectively.
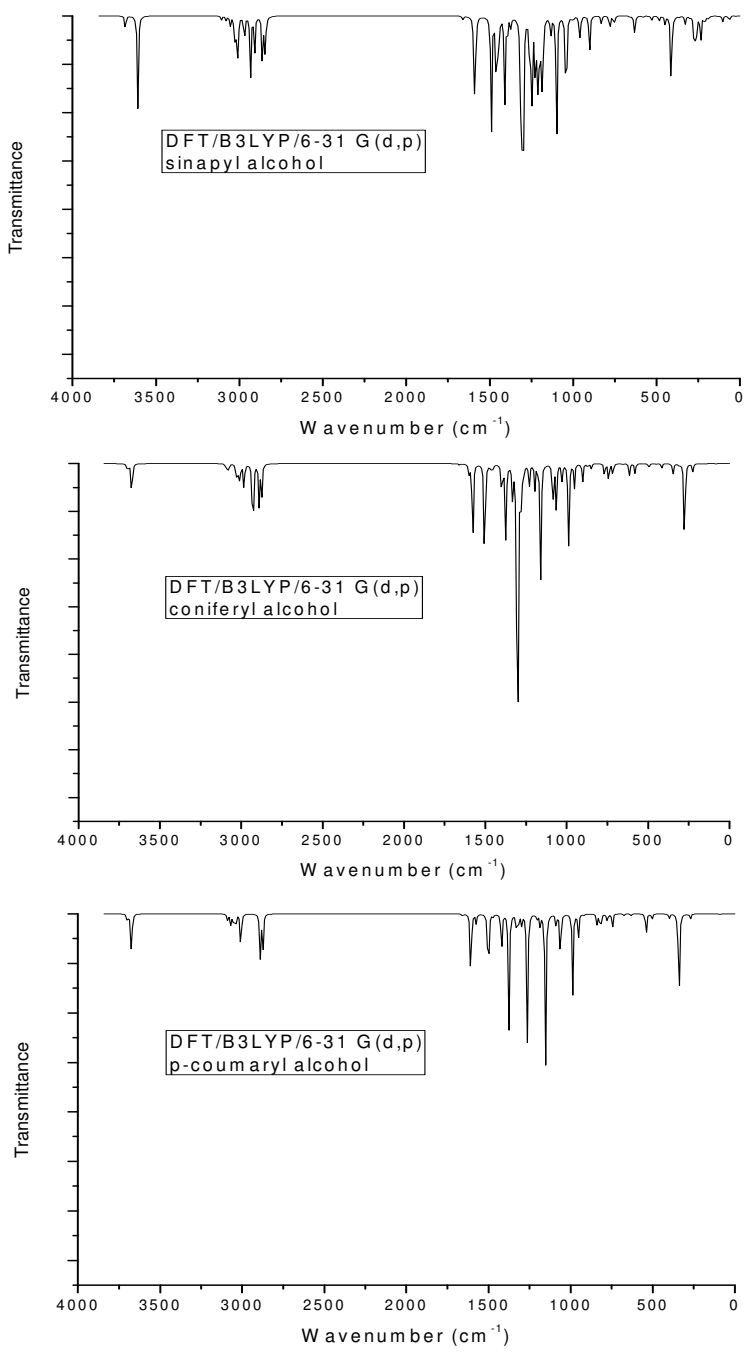

Figure 2: Calculated vibrational frequencies of monolignols 


\section{FERIDE AKMAN}
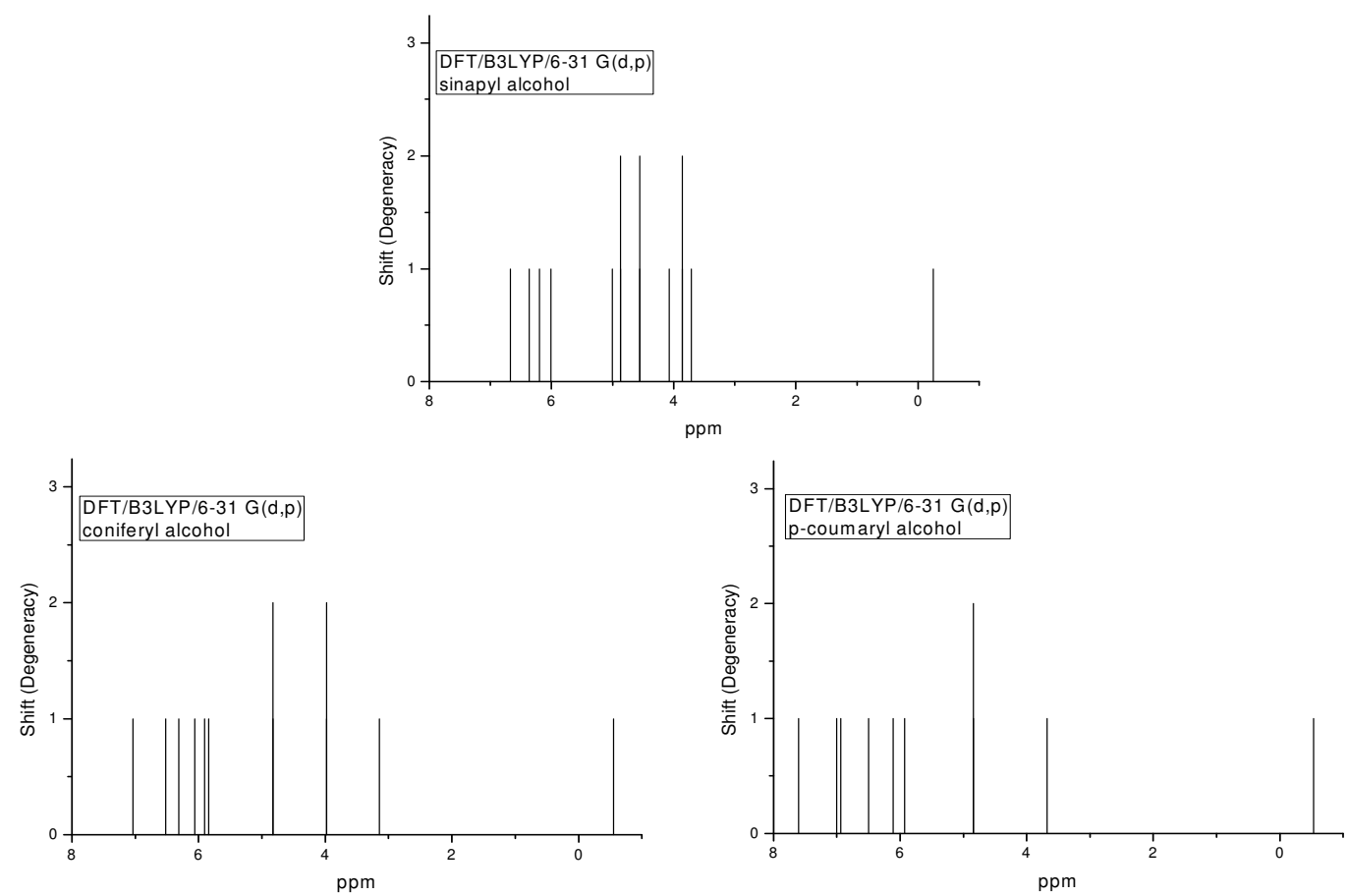

Figure 3: Calculated ${ }^{1} \mathrm{H}-\mathrm{NMR}$ of monolignols

The aromatic ring $\mathrm{C}=\mathrm{C}$ stretching vibrations mostly indicate the region of $1625-1400 \mathrm{~cm}^{-1} \cdot{ }^{17}$ The values computed for the $\mathrm{C}=\mathrm{C}$ stretching signals are in accordance with the experimental values in the literature. Besides, the bands at 1465,1469 and $1469 \mathrm{~cm}^{-1}$ are due to the signal of $\mathrm{CH}_{2}$, next to the ethylene and hydroxyl group. As a result, the theoretical results are compatible with each other.

\section{NMR analysis}

The theoretical ${ }^{1} \mathrm{H}-\mathrm{NMR}$ spectra of the monolignols are indicated in Figure 3. The theoretical ${ }^{1} \mathrm{H}-\mathrm{NMR}$ spectra of sinapyl, coniferyl and p-coumaryl alcohols show characteristic signals at 6.20-6.68 ppm, 6.31-7.04 and 6.50-7.61 $\mathrm{ppm}$, which are ascribed to aromatic ring protons, respectively. The signals at 5.01, 3.14 and 3.68 $\mathrm{ppm}$ are ascribed to $-\mathrm{OH}$ protons bound to the aromatic ring, respectively; while the signals at 6.01 and 6.37, 5.91 and 6.06, 5.93 and $6.11 \mathrm{ppm}$ are ascribed to ethylene protons, respectively. Besides, in sinapyl alcohol, the signals at 3.71$4.56 \mathrm{ppm}$ are ascribed to the protons of methoxy groups bound to the aromatic ring, the signals at $4.87 \mathrm{ppm}$ are ascribed to $-\mathrm{CH}_{2}$ protons adjacent to hydroxyl and ethylene groups. In coniferyl alcohol, the signals at 3.97-5.84 ppm are ascribed to the protons of methoxy groups bound to the aromatic ring, the signals at $4.82 \mathrm{ppm}$ are ascribed to $-\mathrm{CH}_{2}$ protons adjacent to hydroxyl and ethylene groups. In p-coumaryl alcohol, the signals at $4.84 \mathrm{ppm}$ are ascribed to $-\mathrm{CH}_{2}$ protons adjacent to hydroxyl and ethylene groups. The signals in the region between 8.00 and $7.00 \mathrm{ppm}$ are generally ascribed to the chemical shift of aromatic protons. As a result, the theoretical results are compatible with each other.

\section{Frontier molecular orbitals}

LUMO stands for the lowest unoccupied molecular orbital, and HOMO stands for the highest occupied molecular orbital; they are also called FMOs (frontier molecular orbitals). While LUMO acts as an electron acceptor, HOMO acts as an electron donor. The 3D plots of LUMO and HOMO for the monolignols are exhibited in Figure 4. The electronegativity $(\chi)$, electron affinity (A), chemical hardness ( $\eta)$, ionization potential (I), chemical potential $\left(\mu_{o}\right)$, softness $(\varsigma)$ and electrophilicity index $(\omega)$ were calculated with the help of the energy gap between HOMOLUMO, using the B3LYP/6-31G $(\mathrm{d}, \mathrm{p})$ basis set, ${ }^{18}$ and the values are listed in Table 2. 

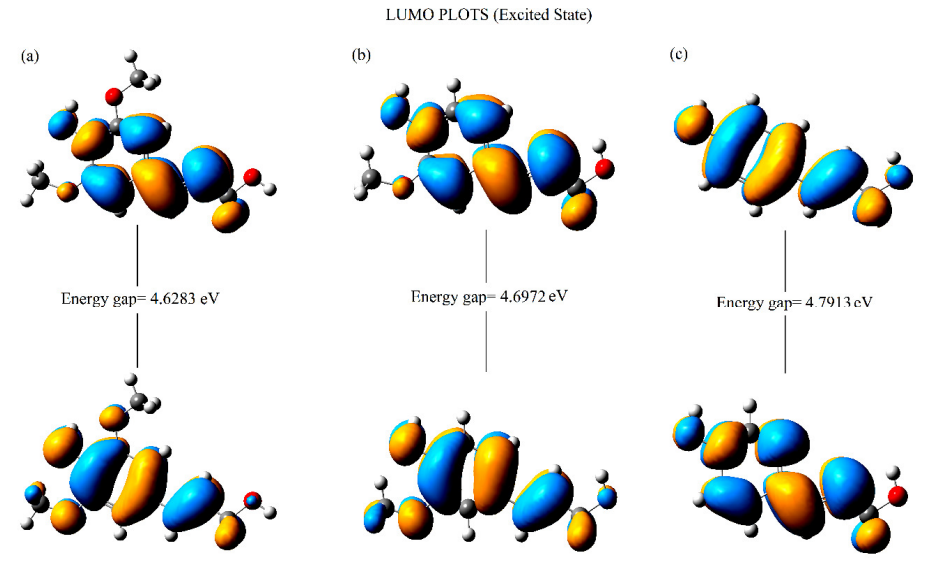

HOMO PLOTS (Ground Statc)

Figure 4: Frontier molecular orbitals (FMOs) of sinapyl alcohol (a), coniferyl alcohol (b) and p-coumaryl alcohol (c)

Table 2

Some electronic properties for monolignols at B3LYP/6-31G $(\mathrm{d}, \mathrm{p})$ basis set in gaseous phase

\begin{tabular}{lccc}
\hline Parameters $(\mathrm{eV})$ & Sinapyl alcohol & Coniferyl alcohol & p-Coumaryl alcohol \\
\hline $\mathrm{E}_{\mathrm{HOMO}}$ & -5.075 & -5.360 & -5.560 \\
$\mathrm{E}_{\mathrm{LUMO}}$ & -0.447 & -0.663 & -0.769 \\
Energy band gap $\left[\Delta \mathrm{E}=\mathrm{E}_{\text {LUMO }}-\mathrm{E}_{\text {HОмо }}\right]$ & 4.628 & 4.697 & 4.791 \\
Electrophilicity index $(\omega)$ & 1.647 & 1.930 & 2.089 \\
Softness $(\varsigma)$ & 0.432 & 0.425 & 0.417 \\
Ionization energy $(\mathrm{I})$ & 5.075 & 5.360 & 5.560 \\
Chemical potential $\left(\mu_{\mathrm{o}}\right)$ & 2.761 & 3.011 & 3.164 \\
Dipole moment $(\mu)$ & 1.537 Debye & 3.776 Debye & 2.538 Debye \\
Electron affinity $(\mathrm{A})$ & 0.447 & 0.663 & 0.769 \\
Electronegativity $(\chi)$ & -2.761 & -3.011 & -3.164 \\
Chemical hardness $(\eta)$ & 2.314 & 2.348 & 2.395 \\
SCF energy & -19822.114 & -16705.456 & -13589.425 \\
\hline
\end{tabular}

(a)

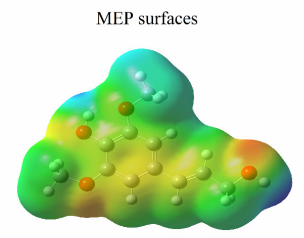

(b)

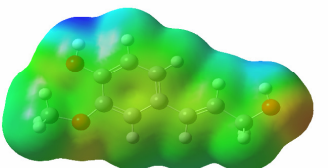

(c)

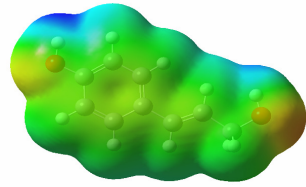

ESP surfaces
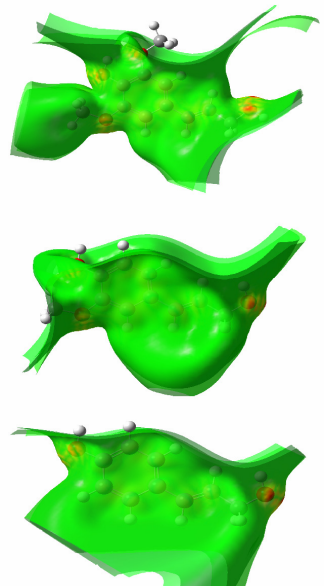

Figure 5: MEP and ESP surfaces of sinapyl alcohol (a), coniferyl alcohol (b) and p-coumaryl alcohol (c)

If the energy gap between HOMO and LUMO is small, then the molecule is associated with high polarisability, low kinetic stability and high chemical reactivity. ${ }^{19}$ In the sinapyl, coniferyl and p-coumaryl alcohols, the value of the HOMOLUMO energy gap was found to be 4.62, 4.69 and 
$4.79 \mathrm{eV}$, respectively, demonstrating that charge transfer occurs to a greater extent within sinapyl alcohols.

\section{Molecular electrostatic potential analysis}

Molecular electrostatic potential (MEP) maps show the electron density of the molecules three dimensionally and serve as very important descriptors for nucleophilic and electrophilic attack regions and hydrogen bonding interactions. ${ }^{20,21}$ Analysis of MEP surfaces was performed by using the same basis set to determine the reactive regions of nucleophilic and electrophilic attack for the monolignols and the results are illustrated in Figure 5. The different colors on the MEP surfaces are the result of different values of electrostatic potential. Potential values decrease in the order of blue $>$ green $>$ yellow $>$ orange $>$ red. The negative region of MEP (red coded region), which is related to electrophilic reactivity, covers the hydroxyl groups due to the oxygen atoms, while the positive region (blue coded region), which is related to nucleophilic reactivity, covers the hydrogen atoms. The higher electronegativity in the hydroxyl groups makes it the most reactive part in the monolignols. The electrostatic potential (ESP) surfaces of monolignols are indicated in Figure 5. The partial charges and electronegativity of the atoms affect the electrostatic potential values. Therefore, the negative ESP is localized more around the oxygen atoms (seen as a reddish blob), whereas the positive ESP is localized on the rest of the monolignols.

\section{CONCLUSION}

In the present study, sinapyl alcohol, pcoumaryl alcohol and coniferyl alcohol were researched theoretically. Firstly, the molecular geometries and molecular structural parameters of the monolignols were determined using the density functional theory (DFT/B3LYP) method, with $6-31 \mathrm{G}(\mathrm{d}, \mathrm{p})$ basis set. Secondly, the scaled vibrational frequencies and the chemical shifts of the monolignols were calculated using the same method. Finally, the analysis of the MEP surfaces was performed to determine the reactive regions and the energy gap between LUMO and HOMO was computed. We hope that this study will be useful to the researchers who are in quest of theoretical and experimental evidence related to the use of monolignols in various materials and applications. Because earlier studies conducted by the DFT method have been confirmed experimentally, future computation using this method can serve as a great tool to scientists.

ACKNOWLEDGEMENTS: The author wishes to thank the Scientific and Technological Application and Research Center of Bingöl University, and Bitlis Eren University for providing the Gaussian software.

\section{REFERENCES}

1 B. M. Upton and A. M. Kasko, Chem. Rev., 116, 2275

(2016),

https://doi.org/10.1021/acs.chemrev.5b00345

2 K. V. Sarkanen and C. H. Ludwig, "Lignins: Occurrence, Formation, Structure and Reactions", Wiley-Interscience, 1971.

3 A. E. Harman-Ware, M. Crocker, A. P. Kaur, M. S. Meier, D. Kato et al., J. Anal. Appl. Pyrol., 99, 161 (2013), https://doi.org/10.1016/j.jaap.2012.10.001

4 D. G. J. Mann, N. L. Labbe, R. W. Sykes, K. Gracom, L. Kline et al., Bioenerg. Res., 2, 246 (2009), https://doi.org/10.1007/s12155-009-9054-X

5 J. R. Obst, Holzforschung, 36, 143 (1982), https://doi.org/10.1515/hfsg.1982.36.3.143

6 J. Zakzeski, P. C. A. Bruijnincx, A. L. Jongerius and B. M. Weckhuysen, Chem. Rev., 110, 3552 (2010), https://doi.org/10.1021/cr900354u

D. Huber, A. Ortner, A. Daxbacher, G. S. Nyanhongo, W. Bauer et al., ACS Sustain. Chem. Eng., 4, 5303 (2016), 10.1021/acssuschemeng.6b00692

F. Akman, Polym. Bull., 74, 2975 (2017), DOI: 10.1007/s00289-016-1875-0

9 F. Akman, Can. J. Phys., 94, 853 (2016), https://doi.org/10.1139/cjp-2016-0252

10 M. J. Frisch, G. W. Trucks, H. B. Schlegel, G. E. Scuseria, M. A. Robb et al., Gaussian 09, Gaussian Inc., Wallingford CT, 2010.

11 R. Dennington, T. Keith and J. Millam, GaussView, Version 5, Semichem Inc., Shawnee Mission KS, 2010.

12 A. D. Becke, Phys. Rev. A, 38, 3098 (1988), https://doi.org/10.1103/PhysRevA.38.3098

13 C. Lee, W. Yang and R. G. Parr, Phys. Rev. B, 37, 785 (1988), https://doi.org/10.1103/PhysRevB.37.785

14 A. D. Becke, J. Chem. Phys., 98, 5648 (1993), https://doi.org/10.1063/1.464304

15 G. Varsanyi, "Assignments for Vibrational Spectra of Seven Hundred Benzene Derivatives", vol. 1, Adam Hilger, London, 1974.

16 R. M. Silverstein and F. X. Webster, "Spectroscopic Identification of Organic Compounds", sixth ed., John Willey and Sons, New York, 1998.

17 V. Krishnakumar, N. Surumbarkuzhali and S. Muthunatesan, Spectrochim. Acta A, 71, 1810 (2009).

18 F. Akman, Cellulose Chem. Technol., 51, 253 (2017),

http://www.cellulosechemtechnol.ro/pdf/CCT34(2017)/p.253-262.pdf 
19 I. Fleming, "Frontier Orbital and Organic Chemical Reactions", John Wiley and Sons, New York, 1976.

20 E. Scrocco and J. Tomasi, Adv. Quantum. Chem., 103, 115 (1978), https://doi.org/10.1016/S00653276(08)60236-1
21 F. J. Luque, J. M. Lopez and M. Orozco, Theor. Chem. Acc., 103, 343 (2000), https://doi.org/10.1007/s002149900013 